\title{
Effects of general anesthesia versus local anesthesia in primary hepatocellular carcinoma patients presenting for thermal ablation surgery: a multiple center retrospective cohort study with propensity score matching
}

\author{
Xiaoqiang Wang ${ }^{1 \#}$, Weiying Xie ${ }^{2 \#}$, Shuyuan Gan ${ }^{3 \#}$, Tao Wang ${ }^{4}$, Xuexin Chen ${ }^{5}$, Diansan Su ${ }^{1}$, Jiaxin Sun ${ }^{6}$, \\ Jiapiao Lin ${ }^{3}$, Feixiang $\mathrm{Wu}^{7}$, Pingbo Xu ${ }^{8}$, Changhong Miao ${ }^{8}$, Min $\mathrm{Yan}^{2}$, Shengmei Zhu ${ }^{3}$, Bo Zhai ${ }^{4}$, \\ Yuming $\operatorname{Sun}^{7}$, Weifeng Yu ${ }^{1}$, Jie Tian ${ }^{1}$
}

${ }^{1}$ Department of Anesthesiology, Renji Hospital, Shanghai Jiaotong University School of Medicine, Shanghai 200127, China; ${ }^{2}$ Department of Anesthesiology, The Second Affiliated Hospital, ${ }^{3}$ Department of Anesthesiology, The First Affiliated Hospital, Zhejiang University School of Medicine, Hangzhou 310006, China; ${ }^{4}$ Department of Interventional Oncology, Renji Hospital, Shanghai Jiaotong University School of Medicine, Shanghai 200127, China; ${ }^{5}$ Department of Anesthesiology, Cancer Hospital, The General Hospital of Ningxia Medical University, Ningxia 750004, China; ${ }^{6}$ Department of Anesthesiology, Sichuan Cancer Hospital \& Institute, Sichuan Cancer Center, School of Medicine, University of Electronic Science and Technology of China, Chengdu 610041, China; ${ }^{7}$ Department of Anesthesiology \& Intensive Care, Eastern Hepatobiliary Hospital, The Nary Military Medical University, Shanghai 200438, China; ${ }^{8}$ Department of Anesthesiology, Fudan University Shanghai Cancer Center, Shanghai, 200032, China

Contributions: (I) Conception and design: Y Sun, W Yu, J Tian; (II) Administrative support: J Tian, D Su, W Yu; (III) Provision of study materials or patients: X Wang, W Xie, S Gan, T Wang, J Sun, J Lin, F Wu, P Xu; (IV) Collection and assembly of data: X Wang, W Xie, S Gan, B Zhai, X Chen; (V) Data analysis and interpretation: X Wang, D Su, C Miao, M Yan, S Zhu, B Zhai, J Tian; (VI) Manuscript writing: All authors; (VII) Final approval of manuscript: All authors.

\#These authors contributed equally to this work.

Correspondence to: Jie Tian. Department of Anesthesiology, Renji Hospital, Shanghai Jiaotong University School of Medicine, Pujian Road 160, Pudong District, Shanghai 200127, China. Email: vaseline2001@hotmail.com; Weifeng Yu. Department of Anesthesiology, Renji Hospital, Shanghai Jiaotong University School of Medicine, Pujian Road 160, Pudong District, Shanghai 200127, China. Email: ywf808@yeah.net; Yuming Sun. Department of Anesthesiology \& Intensive Care, Eastern Hepatobiliary Hospital, the Navy Military Medical University, Changhai Road 225, Yangpu District, Shanghai 200438, China. Email: 13301836930@163.com.

Background: Whether anesthesia methods affect the prognosis of tumor patients is controversial. With the aim of comparing the effects of general anesthesia (GA) and local anesthesia (LA) in primary hepatocellular carcinoma (HCC) patients presenting for elective thermal ablation (TA) surgeries, a multiple center retrospective cohort study was designed and implemented.

Methods: Patients who received elective TA surgery under GA or LA from Jan. 2014 to Dec. 2016 and met the eligibility criteria were included. Survival analysis was used to identify the influence of anesthesia methods on recurrence-free survival (RFS) and overall survival (OS). Propensity score matching (PSM) was used to minimize the bias between the GA group and the LA group.

Results: A total of 244 patients with GA and 245 with LA were eligible for analysis. After PSM, 178 patients remained in each group. In the matched groups, GA showed a significantly higher recurrence rate compared with LA by both the Kaplan-Meier survival analyses $(\mathrm{P}=0.011)$ and multivariable Cox regression analyses $(\mathrm{P}=0.002)$. The multivariable Cox regression model also revealed that $\mathrm{GA}$ had a hazard ratio (HR) of $1.746(\mathrm{P}=0.036)$ for death compared with the LA group.

Conclusions: GA is associated with decreased RFS and OS after surgery compared with LA in HCC patients undergoing TA surgery. Prospective trials exploring the effects of different anesthetic methods on cancer outcome in these patients are warranted. 
Keywords: Hepatocellular carcinoma (HCC); anesthesia methods; thermal ablation (TA); recurrence-free survival (RFS); propensity score matching (PSM)

Submitted Dec 11, 2019. Accepted for publication Feb 21, 2020.

doi: $10.21037 / \mathrm{atm} .2020 .03 .88$

View this article at: http://dx.doi.org/10.21037/atm.2020.03.88

\section{Introduction}

Hepatocellular carcinoma (HCC) ranks as the third leading cause of cancer-related death worldwide (1). Notably, the incidence and mortality rates of HCC in Southeast Asia and Africa are higher than other areas due to the high prevalence of hepatitis virus infection (2). In China, for example, it remains among the top three fatal carcinomas (3).

Recurrence is a critical problem in HCC treatment. Cancer can reoccur in HCC patients at virtually any stage, regardless of the treatments the patients have received $(3,4)$. Even liver transplantation cannot eliminate the recurrence of HCC (5). According to recent studies, the 5-year recurrence rate of HCC ranges from $70 \%$ to $83.6 \%(4,6)$.

Tumor recurrence involves multiple causes and anesthesia methods and anesthetics used have drawn much attention recently. Studies have shown that anesthesia choices may influence the prognosis of tumor patients (7-9). For example, Exadaktylos et al. found that general anesthesia (GA) combined with paravertebral anesthesia and analgesia reduced the risk of recurrence or metastasis, compared with GA combined with postoperative morphine analgesia (7). Two recent studies investigating the association of anesthetics (volatile versus intravenous anesthesia) with long-term survival in patients reported that volatile inhalational agents are associated with lower survival rates $(8,9)$. However, several retrospective studies also demonstrated that perioperative epidurals are not associated with decreased cancer recurrence compared with no epidural use $(10,11)$. More studies are needed to explore effects of anesthesia on the prognosis of cancer patients. Moreover, most previous studies were comparisons between GA and GA combined with regional anesthesia. Whether the differences are due to the negative effects of GA, or the protective effects of regional anesthesia, are unknown.

Although hepatic resection is a standard treatment for HCC, a minimally invasive procedure, thermal ablation (TA), has also been widely used in the clinic. TA includes microwave $(\mathrm{MW})$ ablation and radiofrequency (RF) ablation. In a patient whose tumor size is small, TA surgery can be performed under either GA or local anesthesia (LA). While the former can guarantee that the patients experience no discomfort during surgery, the latter is more economical and speeds up the procedure and the patients' recovery. Both anesthetic techniques are widely used in China, with the decision made based on the consensus between the patients and the treating team.

No data have previously been presented that compare tumor recurrence in patients having TA surgery under GA or LA. Exploration of the association between anesthetic technique (GA vs. LA) with long-term survival in these patients provides a unique model for the determination of whether GA affects tumor outcomes per se. Therefore, we carried out a multi-center retrospective cohort study to compare whether there are any differences in recurrencefree survival (RFS) and overall survival (OS) between the GA group and the LA group of patients presenting for elective TA surgeries for liver cancer. We hypothesized that GA is associated with decreased RFS and OS after surgery compared with LA.

\section{Methods}

\section{Study design}

This was a retrospective cohort study.

\section{Participants}

The ethics committee of the Renji Hospital approved this retrospective study [2018(188)]. Since the current study was only a retrospective review of electronic patient medical records, without the need of any samples from patients or performance of any intervention on the patients, a waiver of written informed consent was applied for and was approved by the local ethical review board. An oral consent was always obtained from the patients or families when the data was collected through contacting them by telephone. This multi-center study was conducted at five university hospitals in China (Renji Hospital Affiliated to Shanghai Jiaotong 
University School of Medicine, Fudan University Shanghai Cancer Center, Eastern Hepatobiliary Surgery Hospital affiliated to the Second Military Medical University, The First Affiliated Hospital of Zhejiang University, and The Second Affiliated Hospital of Zhejiang University). The study was conducted in accordance with the Declaration of Helsinki and is in line with the STROBE criteria. Patients who were aged 18 to 75 years old, presenting for elective TA surgery (including RF ablation and MW ablation) under GA or LA from Jan. 2014 to Dec. 2016 with a diagnosis of primary liver cancer and the summary of the long diameter of all HCC was $\leq 5 \mathrm{~cm}$, were included. Exclusion criteria were as follows: (I) an American Society of Anesthesiologists degree $\geq$ IV or Child-Pugh degree C; (II) a history of any kinds of cancers or was currently diagnosed with any cancers from other organs or systems; (III) a history of liver surgery; (IV) received adjuvant chemoradiotherapy before the ablation surgery; $(\mathrm{V})$ a combined procedure of liver resection surgery and MW/RF ablation surgery; (VI) severe organ failure (heart, pulmonary, hepatic, or renal diseases) or immune system diseases; (VII) distal lymph node or extrahepatic metastasis; (VIII) received liver transplantation surgery after the ablation surgery. HCC diagnoses were confirmed by postoperative histopathology.

Patients in the GA group received propofol, midazolam, fentanyl/sufentanil for anesthesia induction. Muscle relaxant was given when laryngeal mask airway (LMA) insertion and mechanical ventilation was needed. All patients lost consciousness during the procedure. Anesthesiologists were required for sedation, LMA insertion, and hemodynamic monitoring. Some anesthesiologists would give the patients nonsteroidal anti-inflammatory drugs (NSAIDs) before the ablation started to provide preemptive analgesia. GA was maintained with sevoflurane and/or propofol. The patients recovered in Post Anesthesia Care Unit (PACU) and were given intravenous sufentanil or NSAIDs for post-operative pain. In the LA group, less than $10 \mathrm{~mL}$ of $2 \%$ lidocaine was injected subcutaneously into the puncture points, and NSAIDs were used when patients experienced pain, without an anesthesiologist present. Patients were awake and breathed spontaneously during the operation. NSAIDs were used when necessary after surgery.

\section{Variables and data sources}

Over twenty retrospectively collected variables were analyzed, which were categorized as patient characteristics, liver function variables, operative variables and follow- up information. All patients were routinely followed-up after surgery. They were reexamined using serum AFP, ultrasound or CT, and chest X-ray at 1 month after surgery. Patients were then followed-up at 2-month intervals for the first 6 months and at 3-month interval thereafter. Tumor recurrence was defined as new appearance of intraor extrahepatic tumor nodule, with or without a rise in serum AFP. The diagnosis of tumor recurrence refers to the clinical practice guidelines of EASL-EORTC (12). In every center, two trained researchers oversaw data collection and all data was entered using "Excel" or "Epidata".

The timeframe for evaluation ended on March 1st, 2018. The primary outcome of the study was RFS and the second was OS. The RFS was calculated from the date of the ablation surgery to the date of diagnosis of the first recurrence. If no recurrence of tumor was recorded, the RFS was defined as the time between the date of surgery and the date of last follow-up. The survival time was defined as the time between surgery and death. Data were censored for patients who were alive at the follow-up closure date (March 1st, 2018).

\section{Sample size and power}

According to published studies (13), we assumed that the 3 -year recurrence rate of HCC would be approximately $60 \%$ in the GA group and $40 \%$ in the LA group. To achieve a two-sided $\alpha$-level of 0.05 and $90 \%$ power, approximately 255 patients would be needed. Considering that there would be patients lost to follow-up, 284 patients (142 patients per group) were needed if the loss rate was $10 \%$.

\section{Statistical analysis}

Statistical analyses were carried out using the IBM SPSS Statistics 23.0 (SPSS Inc., Armonk, NY, USA). Categorical variables were reported as number (n) or proportion (\%) and continuous variables were expressed as mean \pm standard deviation (SD) or median [25\% interquartile range, $75 \%$ interquartile range]. The Student's $t$-test was used for comparisons of continuous variables. Otherwise, the MannWhitney $U$ test was applied. Categorical variables were compared with the $\chi^{2}$ test with the Yates correction or the Fisher's exact test (when total sample $<40$ or expected frequency $<1$ ).

To identify independent predictive factors of prognosis, univariable and multivariable Cox regression analyses were used. The RFS and OS rates were compared between 


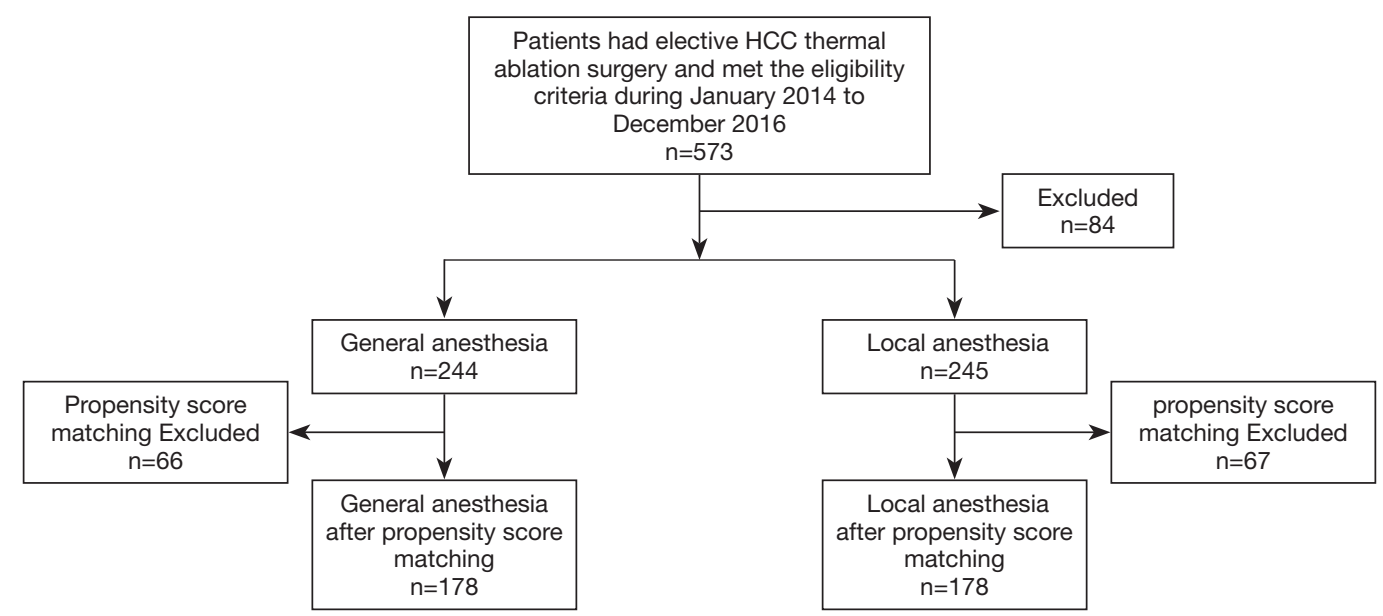

Figure 1 Flow diagram detailing the selection of patients included in the retrospective analysis.

the GA group and LA group using the Kaplan-Meier curves generated by the log-rank test. Multivariable Cox proportional hazard regression analyses were then performed to adjust for other prognostic factors which were associated with OS and RFS (14). Multivariable regression curves were generated as well after multivariable Cox regression analyses.

The propensity score matching (PSM) method, as described before (15), was utilized to eliminate the imbalance in baseline characteristics between the two groups. A logistic regression model was built given the covariates of age, sex, diabetes, cardiopathy, ASA score, tumor number, cirrhosis, HBV/HCV infection, Child-Pugh stage, TNM stage, AFP, ALB, TBIL, ALT, AST, tumor size, adjuvant chemoradiotherapy and hypertension. We applied 1:1 nearest neighbor matching without replacement in order to ensure that conditional bias was minimized. For each patient with GA, a patient having LA with a minimum in distance of propensity scores was matched. To explore the most appropriate caliper width, the caliper width of 0.05 , 0.01 and 0.001 was used for PSM respectively. Finally, results showed that the caliper width of 0.01 met the criteria of both preferable homogeneity and minor loss of sample size. PSM was carried out using IBM SPSS Statistics 23.0 version.

All statistical tests were 2 sided, and $\mathrm{P}$ values $<0.05$ were considered statistically significant.

\section{Results}

All available patients presenting for elective HCC TA surgery, from Jan. 2014 to Dec. 2016, in the five hospitals were screened. Five hundred and seventy-three patients who met the eligibility criteria were enrolled, among which, 84 patients were lost to follow-up. Ultimately, there were 244 patients remaining in the GA group and 245 in the LA group (Figure 1).

The comparisons of patients' baseline characteristics and other variables between the two groups in the entire cohort are illustrated in Table 1. The GA group was significantly older [57.8 (9.3) years] than the LA group [55.9 (10.2) year; $\mathrm{P}=0.035]$. Patients in the GA group were more likely to have had adjuvant chemoradiotherapy, larger tumor size and higher TBIL levels, compared with the LA group. Other baseline variables between two groups showed similar distributions and fine homogeneity. The incidence of postoperative complications (including infection, fever, pain and seroperitoneum) was also comparable and no differences were detected between the two groups.

Firstly, Kaplan-Meier analysis or univariable Cox regression model analysis was utilized to screen variables that had significant influence on the RFS and OS. As shown in Table S1, age, ASA score, hypertension, diabetes, tumor number, cirrhosis, adjuvant chemoradiotherapy, tumor size, TNM stage and anesthesia methods significantly influenced patients' RFS $(\mathrm{P}<0.05)$. Specifically, the Kaplan-Meier survival curves show that LA is associated with significantly improved RFS when compared with GA (Figure $2 \mathrm{~A}$, Tables 2,S1). For OS, our results indicate that it was significantly influenced by cirrhosis, serum ALB levels, Child-Pugh stage, tumor size, and TNM stage, whereas the anesthesia methods had no influence on the OS (Figure 2B, Table S1). 
Table 1 Comparisons of patients' characteristics and other variables between GA group and LA group in the entire cohort

\begin{tabular}{|c|c|c|c|c|c|c|}
\hline Variables & \multicolumn{3}{|c|}{ Before PSM } & \multicolumn{3}{|c|}{ After PSM } \\
\hline Propensity score & $0.54(0.15)$ & $0.46(0.13)$ & 0 & $0.50(0.12)$ & $0.50(0.12)$ & 0.944 \\
\hline \multicolumn{7}{|l|}{ Sex } \\
\hline Male & 202 & 193 & 0.26 & 149 & 144 & 0.487 \\
\hline Age & $57.8(9.3)$ & $55.9(10.2)$ & 0.035 & $56.12(9.86)$ & $57.58(9.40)$ & 0.155 \\
\hline$\leq 60$ & 147 & 156 & 0.435 & 111 & 113 & 0.826 \\
\hline$>60$ & 97 & 89 & & 67 & 65 & \\
\hline \multicolumn{7}{|l|}{ ASA score } \\
\hline Hypertension (yes/no) & $62 / 182$ & $56 / 189$ & 0.509 & $38 / 140$ & $40 / 138$ & 0.798 \\
\hline Diabetes (yes/no) & $29 / 215$ & $38 / 207$ & 0.244 & 23/155 & $21 / 157$ & 0.747 \\
\hline Cardiopathy $^{\dagger}$ (yes/no) & $10 / 234$ & $13 / 232$ & 0.528 & $6 / 172$ & $6 / 172$ & 1 \\
\hline Tumor number & $1.2(0.5)$ & $1.2(0.4)$ & 0.464 & $1.21(0.46)$ & $1.16(0.40)$ & 0.271 \\
\hline Solitary & 201 & 207 & 0.53 & 144 & 151 & 0.325 \\
\hline Multiple & 43 & 38 & & 34 & 27 & \\
\hline Cirrhosis (yes/no) & $197 / 47$ & $188 / 57$ & 0.279 & $141 / 37$ & $139 / 39$ & 0.796 \\
\hline HBV/HCV infection (yes/no) & $209 / 35$ & $213 / 32$ & 0.68 & $153 / 25$ & $150 / 28$ & 0.655 \\
\hline$<3 \mathrm{~cm}$ & 134 & 164 & 0.006 & 104 & 108 & 0.666 \\
\hline$\geq 3 \mathrm{~cm}$ & 110 & 81 & & 74 & 70 & \\
\hline \multicolumn{7}{|l|}{ TNM stage } \\
\hline I & 197 & 198 & 0.982 & 140 & 148 & 0.281 \\
\hline II & 47 & 47 & & 38 & 30 & \\
\hline AFP* & $16.9[4.6,168.3]$ & $15.7[3.7,167.1]$ & 0.444 & $17.0[5.3,157.5]$ & $13.8[3.5,129.4]$ & 0.18 \\
\hline$<400 \mathrm{ng} / \mathrm{mL}$ & 204 & 211 & 0.438 & 153 & 151 & 0.764 \\
\hline$\geq 400 \mathrm{ng} / \mathrm{mL}$ & 40 & 34 & & 25 & 27 & \\
\hline TBIL & $16.6[12.0,24.0]$ & $14.6[10.9,21.9]$ & 0.02 & $16.6[11.8,24.1]$ & $14.4[10.5,21.3]$ & 0.026 \\
\hline$<34 \mathrm{mmol} / \mathrm{L}$ & 219 & 224 & 0.526 & 161 & 159 & 0.725 \\
\hline$\geq 34 \mathrm{mmol} / \mathrm{L}$ & 25 & 21 & & 17 & 19 & \\
\hline
\end{tabular}

Table 1 (continued) 
Table 1 (continued)

\begin{tabular}{|c|c|c|c|c|c|c|}
\hline Variables & \multicolumn{3}{|c|}{ Before PSM } & \multicolumn{3}{|c|}{ After PSM } \\
\hline ALB & $40.5(6.1)$ & $40.7(4.9)$ & 0.658 & $41.0(5.8)$ & $40.1(5.0)$ & 0.104 \\
\hline$\leq 35 \mathrm{~g} / \mathrm{L}$ & 48 & 31 & 0.035 & 30 & 29 & 0.887 \\
\hline$>35 \mathrm{~g} / \mathrm{L}$ & 196 & 214 & & 148 & 149 & \\
\hline$<40 \mathrm{U} / \mathrm{L}$ & 152 & 146 & 0.54 & 112 & 116 & 0.659 \\
\hline$\geq 40 \mathrm{U} / \mathrm{L}$ & 92 & 99 & & 66 & 62 & \\
\hline AST & $34.7[26.3,61.8]$ & $33.0[22.1,56.5]$ & 0.162 & $34.4[25.0,58.0]$ & $33.0[22.4,54.3]$ & 0.383 \\
\hline$<40 \mathrm{U} / \mathrm{L}$ & 143 & 145 & 0.897 & 106 & 106 & 1 \\
\hline Fever & 8 & 13 & 0.269 & 3 & 11 & 0.053 \\
\hline Pain & 10 & 7 & 0.454 & 7 & 5 & 0.557 \\
\hline Seroperitoneum & 2 & 1 & 0.623 & 2 & 0 & 0.499 \\
\hline Infection & 1 & 0 & 0.499 & 1 & 0 & 1 \\
\hline
\end{tabular}

Variables are shown as "mean (SD)" or "median [25\% quartile, 75\% quartile]". ${ }^{\dagger}$, cardiopathy illnesses include coronary heart disease, heart failure (NYHA I-II), arrhythmia, myocardiopathy and valvulopathy; ${ }^{\ddagger}$, adjuvant chemoradiotherapy includes transcatheter arterial chemoembolization (TACE) and radioactive seed implantation. Adjuvant chemoradiotherapy is defined as patients received TACE or radioactive seed implantation simultaneously with or after TA surgery; ${ }^{\S}$, the number of patients who had postoperative complications is sometimes smaller than the summary of patients' number with individual complications because some patients had more than one complication. ASA, American Society of Anesthesiologists; HBV, hepatitis B virus; HCV, hepatitis C virus; TNM, Clinicopathological stage; AFP, alpha-fetoprotein; TBIL, total bilirubin; ALB, serum albumin; ALT, Alanine transaminase; AST, aspartate aminotransferase; SD, standard deviation; GA, general anesthesia; LA, local anesthesia.

Significant variables $(\mathrm{P}<0.05)$ as shown in Table $S 1$ were entered into multivariable Cox regression model analysis. Again, the presence of postoperative recurrence was significantly lower in the LA group than in the GA group $(\mathrm{P}=0.034)$, although no statistical difference was found in OS $(\mathrm{P}>0.05)$ between two anesthesia methods, as shown in Table 3 and Figure 2C,D, indicating that LA was a better choice for HCC patients undergoing TA surgery.

PSM analysis was carried out as illustrated in Methods and created 178 pairs of patients. After the PSM, there were no significant differences in the baseline characteristics between the two groups (Table 1). GA was still shown to be associated with a significantly increased hazard ratio (HR) in both univariable [1.445 (95\% CI, 1.088-1.921)] and multivariable [1.593 (95\% CI, 1.193-2.127)] Cox regression analysis in RFS (Tables S1,3). The means of RFS in the PS-matched GA group and LA group were 755.2 and 928.6 days, respectively.
Notably, when the balance between the two groups was significantly improved by matching, patients who received GA also exhibited significantly worse OS than those who received LA by multivariable Cox regression analysis [with an HR of 1.746 (95\% CI, 1.036-2.943)] (Table 3). The means of OS in the PS-matched GA group and LA group were $1,277.5$ and 1,365.2 days, respectively. Survival curves are shown in Figure 3A,B,C,D. Besides anesthesia methods, TNM stage and tumor size were independent risk factors for both RFS and OS (Table 3). Variables that significantly deteriorated RFS but not OS after the multivariable analysis were adjuvant chemoradiotherapy, cirrhosis, higher ASA score and lower ALB levels (Table 3).

\section{Discussion}

In this multi-center retrospective cohort study, we found 



Figure 2 The survival analyses of RFS and OS before PSM. (A) Kaplan-Meier RFS curves, (B) Kaplan-Meier OS curves, (C) multivariable Cox survival analysis RFS curves, and (D) multivariable Cox survival analysis OS curves by anesthesia methods. RFS, recurrence-free survival; OS, overall survival.

Table 2 The RFS rates of patients in GA group and LA group

\begin{tabular}{|c|c|c|c|c|c|c|c|c|c|}
\hline Time & \multicolumn{4}{|c|}{ GA group $(n=244)$} & \multicolumn{4}{|c|}{ LA group $(n=245)$} & $-P$ value \\
\hline At treatment & 100 & & & 244 & 100 & & & 245 & \\
\hline 1 year & $56.1(49.8-62.4)$ & 107 & 0 & 137 & $71.4(65.7-77.1)$ & 70 & 0 & 175 & $<0.01$ \\
\hline 2 years & $45.0(38.7-51.3)$ & 132 & 39 & 73 & $55.1(48.8-61.4)$ & 108 & 25 & 112 & $<0.05$ \\
\hline 4 years & $30.9(19.5-42.3)$ & 143 & 100 & 1 & $44.2(37.3-51.1)$ & 126 & 88 & 31 & $<0.05$ \\
\hline
\end{tabular}

*, point-wise $95 \% \mathrm{Cl}$. RFS, recurrence-free survival; Cl, confidence interval; GA, general aneashtesia; LA, local aneashtesia.

that anesthesia methods significantly influenced the longterm survival of HCC patients after TA surgery. After PSM and adjustment for known confounding factors, GA had an HR of $1.593(\mathrm{P}=0.002)$ for cancer recurrence and 1.746
$(\mathrm{P}=0.036)$ for death in a multivariable Cox regression model compared with LA. These findings indicate that LA might a better choice compared with GA for patients who require TA surgery for HCC. 
Table 3 Multivariable Cox regression model analysis of RFS and OS

\begin{tabular}{|c|c|c|c|}
\hline Independent predictive factor & $\mathrm{HR}$ & $95 \% \mathrm{Cl}$ & $P$ value \\
\hline \multicolumn{4}{|l|}{ RFS (before PSM) } \\
\hline \multicolumn{4}{|l|}{ Anesthesia methods } \\
\hline LA & 1 & & \\
\hline GA & 1.314 & $1.021-1.691$ & 0.034 \\
\hline \multicolumn{4}{|l|}{ TNM stage } \\
\hline I & 1 & & \\
\hline ॥ & 2.924 & $1.558-5.487$ & 0.001 \\
\hline \multicolumn{4}{|l|}{ Tumor size } \\
\hline$<3 \mathrm{~cm}$ & 1 & & \\
\hline$\geq 3 \mathrm{~cm}$ & 1.356 & $1.043-1.762$ & 0.023 \\
\hline \multicolumn{4}{|l|}{ Adjuvant chemoradiotherapy } \\
\hline No & 1 & & \\
\hline Yes & 1.531 & $1.163-2.016$ & 0.002 \\
\hline \multicolumn{4}{|l|}{ Cirrhosis } \\
\hline No & 1 & & \\
\hline Yes & 1.428 & $1.027-1.985$ & 0.034 \\
\hline \multicolumn{4}{|l|}{ Hypertension } \\
\hline No & 1 & & \\
\hline Yes & 1.238 & $0.927-1.652$ & 0.149 \\
\hline \multicolumn{4}{|l|}{ ASA score } \\
\hline II & 1 & & \\
\hline III & 1.295 & $0.935-1.792$ & 0.12 \\
\hline \multicolumn{4}{|l|}{ Age } \\
\hline$\leq 60$ & 1 & & \\
\hline$>60$ & 1.162 & $0.901-1.497$ & 0.247 \\
\hline \multicolumn{4}{|l|}{ Tumor number } \\
\hline Solitary & 1 & & \\
\hline Multiple & 1.363 & $0.981-1.894$ & 0.065 \\
\hline \multicolumn{4}{|l|}{ Diabetes } \\
\hline No & 1 & & \\
\hline Yes & 1.125 & $0.790-1.601$ & 0.515 \\
\hline \multicolumn{4}{|l|}{ RFS (after PSM) } \\
\hline \multicolumn{4}{|l|}{ Anesthesia methods } \\
\hline LA & 1 & & \\
\hline GA & 1.593 & $1.193-2.127$ & 0.002 \\
\hline
\end{tabular}

Table 3 (continued)
Table 3 (continued)

\begin{tabular}{|c|c|c|c|}
\hline Independent predictive factor & $\mathrm{HR}$ & $95 \% \mathrm{Cl}$ & $P$ value \\
\hline \multicolumn{4}{|l|}{ TNM stage } \\
\hline 1 & 1 & & \\
\hline II & 2.72 & $1.099-6.728$ & 0.03 \\
\hline \multicolumn{4}{|l|}{ Tumor size } \\
\hline$<3 \mathrm{~cm}$ & 1 & & \\
\hline$\geq 3 \mathrm{~cm}$ & 1.513 & $1.119-2.045$ & 0.007 \\
\hline \multicolumn{4}{|l|}{ Adjuvant chemoradiotherapy } \\
\hline No & 1 & & \\
\hline Yes & 1.838 & $1.308-2.581$ & 0 \\
\hline \multicolumn{4}{|l|}{ Cirrhosis } \\
\hline No & 1 & & \\
\hline Yes & 1.59 & $1.073-2.357$ & 0.021 \\
\hline \multicolumn{4}{|l|}{ ASA score } \\
\hline II & 1 & & \\
\hline III & 1.533 & $1.085-2.165$ & 0.015 \\
\hline \multicolumn{4}{|l|}{ ALB } \\
\hline$>35 \mathrm{~g} / \mathrm{L}$ & 1 & & \\
\hline$\leq 35 \mathrm{~g} / \mathrm{L}$ & 1.458 & $1.018-2.089$ & 0.04 \\
\hline \multicolumn{4}{|l|}{ Tumor number } \\
\hline Solitary & 1 & & \\
\hline Multiple & 1.484 & $0.588-3.745$ & 0.404 \\
\hline \multicolumn{4}{|l|}{ OS (before PSM) } \\
\hline \multicolumn{4}{|l|}{ Anesthesia methods } \\
\hline LA & 1 & & \\
\hline GA & 1.357 & $0.865-2.128$ & 0.184 \\
\hline \multicolumn{4}{|l|}{ TNM stage } \\
\hline I & 1 & & \\
\hline ॥ & 1.744 & $1.051-2.897$ & 0.032 \\
\hline \multicolumn{4}{|l|}{ Tumor size } \\
\hline$<3 \mathrm{~cm}$ & 1 & & \\
\hline$\geq 3 \mathrm{~cm}$ & 1.64 & $1.016-2.647$ & 0.043 \\
\hline \multicolumn{4}{|l|}{ ALB } \\
\hline$>35 \mathrm{~g} / \mathrm{L}$ & 1 & & \\
\hline$\leq 35 \mathrm{~g} / \mathrm{L}$ & 1.483 & $0.810-2.713$ & 0.201 \\
\hline
\end{tabular}

Table 3 (continued) 
Table 3 (continued)

\begin{tabular}{|c|c|c|c|}
\hline Independent predictive factor & $\mathrm{HR}$ & $95 \% \mathrm{Cl}$ & $P$ value \\
\hline \multicolumn{4}{|l|}{ Child-Pugh stage } \\
\hline A & 1 & & \\
\hline B & 1.221 & $0.572-2.607$ & 0.605 \\
\hline \multicolumn{4}{|l|}{ Cirrhosis } \\
\hline No & 1 & & \\
\hline Yes & 1.75 & $0.896-3.420$ & 0.101 \\
\hline \multicolumn{4}{|l|}{ OS (after PSM) } \\
\hline \multicolumn{4}{|l|}{ Anesthesia methods } \\
\hline LA & 1 & & \\
\hline GA & 1.746 & $1.036-2.943$ & 0.036 \\
\hline \multicolumn{4}{|l|}{ TNM stage } \\
\hline I & 1 & & \\
\hline II & 1.965 & $1.078-3.583$ & 0.028 \\
\hline \multicolumn{4}{|l|}{ Tumor size } \\
\hline$<3 \mathrm{~cm}$ & 1 & & \\
\hline$\geq 3 \mathrm{~cm}$ & 1.847 & $1.064-3.205$ & 0.029 \\
\hline \multicolumn{4}{|l|}{ ALB } \\
\hline$>35 \mathrm{~g} / \mathrm{L}$ & 1 & & \\
\hline$\leq 35 \mathrm{~g} / \mathrm{L}$ & 1.913 & $0.964-3.798$ & 0.064 \\
\hline \multicolumn{4}{|l|}{ Child-Pugh stage } \\
\hline A & 1 & & \\
\hline B & 1.672 & $0.723-3.868$ & 0.23 \\
\hline
\end{tabular}

RFS, recurrence-free survival; OS, overall survival; HR, hazard ratio; $\mathrm{Cl}$, confidence interval; TNM, Clinicopathological stage; ASA, American Society of Anesthesiologists; ALB, serum albumin; LA, local anesthesia; GA, general anesthesia; PSM, propensity score matching.

Whether anesthetic drugs and anesthesia methods will influence the prognosis of cancer patients has been a topic of interest in recent years. Previously, numerous retrospective clinical studies show that cancer patients who receive GA combined with regional anesthesia [epidural anesthesia (EA) or peripheral nerve block] at the time of surgery have better prognosis compared with simple GA $(7,8)$. Specifically, laboratory studies with individual anesthetic drugs indicated that propofol, midazolam and local anesthetics may have potential "anti-cancer" properties; whereas inhalational agents and opioids may increase cancer malignancy $(16,17)$.
The potential mechanisms include influencing the body's immune system (18-20), the malignant potential of tumor cells $(16,17,21)$, and the generation of tumor vessels (22). Therefore, the difference between GA combined with regional anesthesia and simple GA may be possibly due to a negative impact of GA, or a protective effect of regional anesthesia, which remains to be elucidated. How utilization of a combination of both beneficial and deleterious anesthetic drugs to induce loss of consciousness in patients per se would affect tumor outcome remains unknown.

In the current study, patients with a diagnosis of primary liver cancer, whose tumor sizes were small enough to tolerate TA surgery under LA, were recruited. We excluded subjects who have had liver surgeries to avoid any influence from previous surgeries or anesthesia, and we determined that there was no metastatic history in both groups. Due to the strict eligibility criteria, only 573 patients were ultimately recruited. The specific selection criteria help to objectively reflect the treatment effects of GA. For the total patient group, multivariable analysis showed that GA was associated with a worse RFS, and in the propensity-matched groups, GA was demonstrated to have deleterious impact on both RFS and OS. This is the first clinical study providing evidence that GA may lead to worse long-term outcomes in HCC TA surgery patients compared with a less than $10 \mathrm{~mL}$ dose of subcutaneous lidocaine.

Previously, Lai and colleagues reported that treatment of small HCC by RF under GA had a lower risk of cancer recurrence when compared with EA (13). In another recent retrospective study, Kuo and colleagues showed that the 2-year OS and RFS rates were not significantly different between the GA group and the non-GA group in HCC patients who underwent RF treatment (23). There are several possible reasons for the inconsistencies between the current study and previous reports. The eligible criteria are stricter and the sample size is larger in our study. More importantly, the anesthetic groups are different among these studies. While Lai and colleagues compared GA with EA, Kuo and colleagues compared GA with non-GA, which included both EA and LA. Moreover, in both studies, patients in the EA or non-GA groups received fentanyl, morphine, or Demerol peri-operatively, all of which belong to opioid family. As opioids are now generally accepted to promote tumor progression and inhibit the immune system $(21,24)$, use of opioids may be a confounding factor.

Several potential mechanisms may explain why GA is associated with increased recurrence and death after TA surgery in HCC patients. First, the body's immune system 

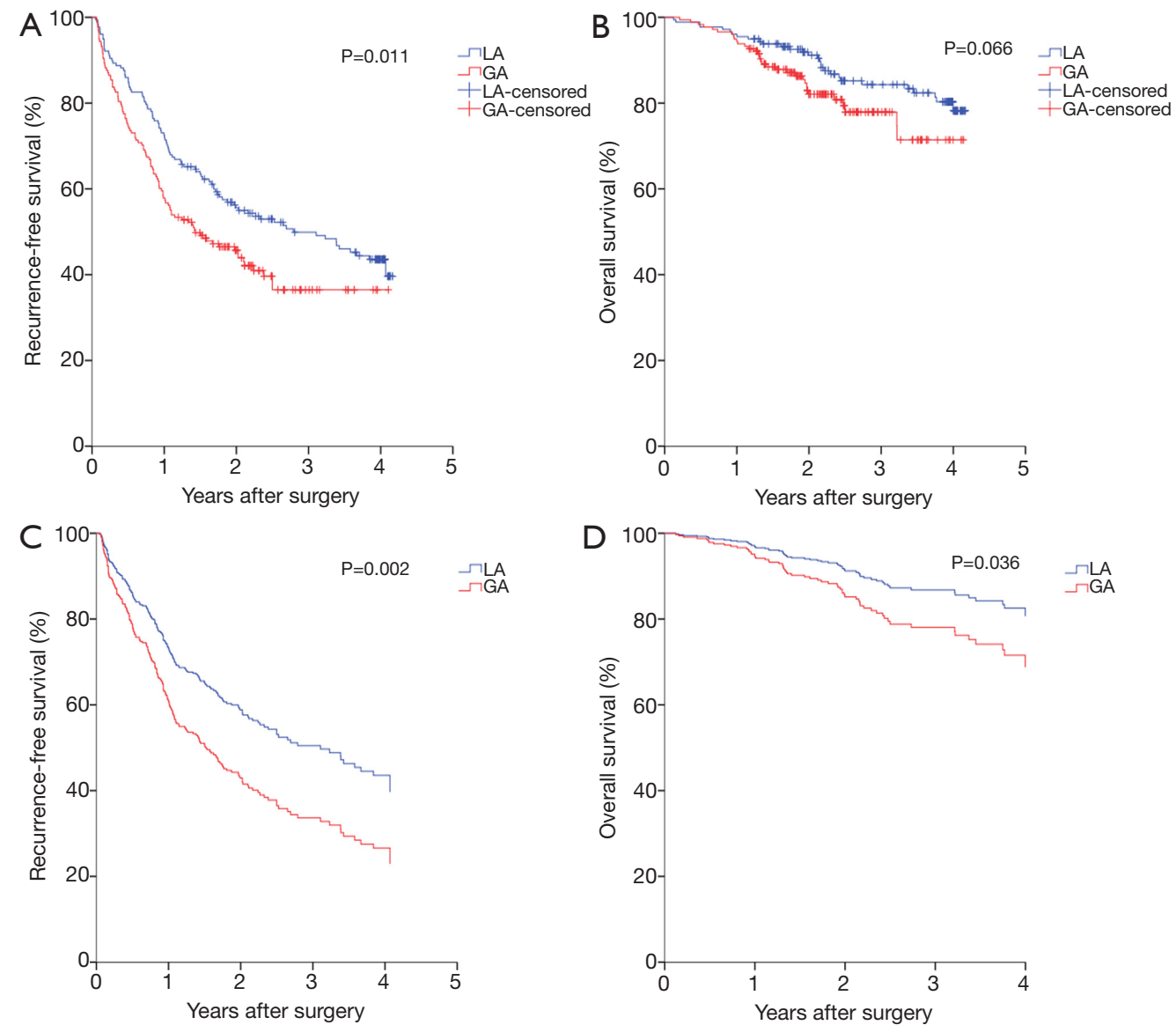

Figure 3 The survival analyses of RFS and OS after PSM. (A) Kaplan-Meier RFS curves, (B) Kaplan-Meier OS curves, (C) multivariable Cox survival analysis RFS curves, and (D) multivariable Cox survival analysis OS curves by anesthesia method after PSM. RFS, recurrencefree survival; OS, overall survival; PSM, propensity score matching.

is generally inhibited after GA (18-20). Many studies have shown that the functions of multiple immune cells, including natural killer cells, effector T cells, lymphocytes, dendritic cells and B cells, are suppressed after GA $(22,23)$. Specifically, inhalational hypnotics and opioids may activate genes related to apoptosis and promote the apoptosis of immune cells $(19,20)$. Additionally, synthesis of inflammatory factors (i.e., IL- $1 \beta$, TNF- $\alpha$ and IL-6) and cytokines (i.e., IFN- $\gamma$, IL-4 and IL-10) is perturbed when patients receive GA, which shifts the balance of Th1/Th2 toward anti-cell-mediated immunity (CMI) Th2 dominance $(22,23)$. Moreover, the effects could be indirect, due to activation of hypothalamic-pituitaryadrenal axis and the sympathetic nervous system. During GA, the neuroendocrine system together with both proinflammatory and anti-inflammatory cytokines augment their immune-suppressive effects. Second, by promoting systemic inflammatory responses, many anesthetics used during GA will subsequently increase the synthesis of vascular endothelial growth factor, cyclooxygenase-2, hypoxia-inducible factor and matrix metalloproteinase, which ultimately stimulate the proliferation and migration capacities of tumor cells and increase stromal angiogenesis $(16,17,21,22,25)$. Although some hypnotic agents, such as propofol and midazolam, may potentially decrease cancer malignancy (25), results from the current study indicate that the general neuroendocrine milieu induced by GA, together with the combined effects from different anesthetics, seem to favor cancer.

In the present study, significantly more patients in the GA group received NSAIDs during perioperative period compared with those in the LA group $(59.8 \%$ vs. $9.0 \%$, $\mathrm{P}<0.0001)$. This is probably because some anaesthesiologists would routinely give patients NSAIDs before the ablation 
starts to provide preemptive analgesia during their GA as long as no contraindications exist. Interestingly, when univariable Cox regression model was performed to analyze the influence of NSAIDs on the study endpoints, we found that it was not associated with either RFS or OS, indicating that use of NSAIDs did not affect the patients' long-term outcome in these HCC patients. Result from the literature about the association between NSAIDs intake and the longterm outcome in the oncological surgical population has been controversial to date, with some studies reporting a beneficial effect, some finding no effect, and some even demonstrating an adverse effect of NSAIDs on patient outcome after cancer surgery (26-28). Researchers postulated that the effects of NSAIDs on survival in the cancer patients depend on the dosage and the duration of treatment and the patients' BMI $(29,30)$. Since majority of the HCC patients in China have low or normal body weight, with a BMI of less than 25 , it is possible that the short-term use of NSAIDs in these patients would not affect the patients' long-term survival outcomes. Further studies are needed to better clarify whether the use of perioperative NSAIDs affects survival.

The present study has some limitations. First, it is a retrospective cohort study. Choice of anesthesia methods was per the surgeons' and patients' preference. Therefore, some of the baseline characteristics differed between the two groups. Although PSM was performed to correct these factors, it cannot be ruled out that some of the unmeasured variables may be confounding factors. In addition, the recurrence date being recorded as the date when follow-up examinations showed abnormities, the recurrence time period depended on the frequency of follow-up and there may be some variations from the actual recurrence time. Second, several patients who met the eligibility criteria had to be excluded because of incomplete data in their medical records. Third, we could not calculate the accurate amounts of specific anesthetic drugs and adjuvant chemotherapeutic drugs, because some of the records did not have exact drug dosage, especially with the old medical record software. However, the names of the drugs used were all accurately recorded. Fourth, the median follow-up time in the current study is 965 days, which is not a long-term followup for tumor studies. Considering the fact that the peak time of HCC recurrence is $1-2$ years (12), this study is nonetheless clinically relevant. Long-term follow-up will yield more precise data.

\section{Conclusions}

In summary, our present study showed that the anesthesia method significantly influenced the prognosis of HCC patients who had TA surgery. Compared with GA, patients who received LA showed a lower rate of tumor recurrence and longer OS after PSM and multivariable Cox regression analysis. This study suggests that prospective trials exploring the effects of LA on cancer outcome in HCC TA surgery patients are warranted.

\section{Acknowledgments}

We thank Dr. Weituo Zhang for statistical support and Dr. Catherine Ketcham for English language editing.

Funding: This research was funded by National Natural Science Foundation of China, grant number 81571048 \& 81571030, Shanghai Science and Technology Committee Foundation, grant number 19ZR1430600, Shanghai Municipal Health Commission Foundation, grant number ZY (2018-2020)-ZYJS-48 and Renji Hospital Clinical Innovation Foundation, grant number PYMDT-006.

\section{Footnote}

Conflicts of Interest: The authors have no conflicts of interest to declare.

Ethical Statement: The authors are accountable for all aspects of the work in ensuring that questions related to the accuracy or integrity of any part of the work are appropriately investigated and resolved. The ethics committee of the Renji Hospital approved this retrospective study [2018(188)].

Open Access Statement: This is an Open Access article distributed in accordance with the Creative Commons Attribution-NonCommercial-NoDerivs 4.0 International License (CC BY-NC-ND 4.0), which permits the noncommercial replication and distribution of the article with the strict proviso that no changes or edits are made and the original work is properly cited (including links to both the formal publication through the relevant DOI and the license). See: https://creativecommons.org/licenses/by-nc-nd/4.0/.

\section{References}

1. Allemani C, Matsuda T, Di Carlo V, et al. Global surveillance of trends in cancer survival 2000-14 (concord-3): Analysis of individual records for 37513 025 patients diagnosed with one of 18 cancers from 
322 population-based registries in 71 countries. Lancet (London, England) 2018;391:1023-75.

2. Pimpin L, Cortez-Pinto H, Negro F, et al. Burden of liver disease in europe: Epidemiology and analysis of risk factors to identify prevention policies. J Hepatol 2018;69:718-35.

3. Bertuccio P, Turati F, Carioli G, et al. Global trends and predictions in hepatocellular carcinoma mortality. $\mathrm{J}$ Hepatol 2017;67:302-9.

4. Colecchia A, Schiumerini R, Cucchetti A, et al. Prognostic factors for hepatocellular carcinoma recurrence. World J Gastroenterol 2014;20:5935-50.

5. Pinna AD, Yang T, Mazzaferro V, et al. Liver transplantation and hepatic resection can achieve cure for hepatocellular carcinoma. Ann Surg 2018;268:868-75.

6. Tian X, Dai Y, Wang DQ, et al. Transarterial chemoembolization versus hepatic resection in hepatocellular carcinoma treatment: A meta-analysis. Drug Des Devel Ther 2015;9:4431-40.

7. Exadaktylos AK, Buggy DJ, Moriarty DC, et al. Can anesthetic technique for primary breast cancer surgery affect recurrence or metastasis? Anesthesiology 2006;105:660-4.

8. Wigmore TJ, Mohammed K, Jhanji S. Long-term survival for patients undergoing volatile versus iv anesthesia for cancer surgery: A retrospective analysis. Anesthesiology 2016;124:69-79.

9. Wu ZF, Lee MS, Wong CS, et al. Propofol-based total intravenous anesthesia is associated with better survival than desflurane anesthesia in colon cancer surgery. Anesthesiology 2018;129:932-41.

10. Cummings KC 3rd, Xu F, Cummings LC, et al. A comparison of epidural analgesia and traditional pain management effects on survival and cancer recurrence after colectomy. Anesthesiology 2012;116:797-806.

11. Myles PS, Peyton P, Silbert B, et al. Perioperative epidural analgesia for major abdominal surgery for cancer and recurrence-free survival: Randomised trial. BMJ (Clinical research ed) 2011;342:d1491.

12. European Association For The Study Of The L, European Organisation For R, Treatment Of C. Easleortc clinical practice guidelines: Management of hepatocellular carcinoma. J Hepatol 2012;56:908-43.

13. Lai R, Peng $Z$, Chen D, et al. The effects of anesthetic technique on cancer recurrence in percutaneous radiofrequency ablation of small hepatocellular carcinoma. Anesth Analg 2012;114:290-6.

14. Yang T, Lu JH, Lau WY, et al. Perioperative blood transfusion does not influence recurrence-free and overall survivals after curative resection for hepatocellular carcinoma: A propensity score matching analysis. J Hepatol 2016;64:583-93.

15. Baek S, Park SH, Won E, et al. Propensity score matching: A conceptual review for radiology researchers. Korean J Radiol 2015;16:286-96.

16. Iwasaki M, Zhao H, Jaffer T, et al. Volatile anaesthetics enhance the metastasis related cellular signalling including cxcr 2 of ovarian cancer cells. Oncotarget 2016;7:26042-56.

17. Yang HF, Yu M, Jin HD, et al. Fentanyl promotes breast cancer cell stemness and epithelial-mesenchymal transition by upregulating alpha1, 6-fucosylation via wnt/beta-catenin signaling pathway. Front Physiol 2017;8:510.

18. Liu S, Wang B, Li S, et al. Immune cell populations decrease during craniotomy under general anesthesia. Anesth Analg 2011;113:572-7.

19. Stollings LM, Jia LJ, Tang P, et al. Immune modulation by volatile anesthetics. Anesthesiology 2016;125:399-411.

20. Anderson SL, Duke-Novakovski T, Singh B. The immune response to anesthesia: Part 1. Vet Anaesth Analg 2014;41:113-26.

21. Niu DG, Peng F, Zhang W, et al. Morphine promotes cancer stem cell properties, contributing to chemoresistance in breast cancer. Oncotarget 2015;6:3963-76.

22. Benzonana LL, Perry NJ, Watts HR, et al. Isoflurane, a commonly used volatile anesthetic, enhances renal cancer growth and malignant potential via the hypoxia-inducible factor cellular signaling pathway in vitro. Anesthesiology 2013;119:593-605.

23. Kuo YH, Chung KC, Hung CH, et al. The impact of general anesthesia on radiofrequency ablation of hepatocellular carcinoma. Kaohsiung J Med Sci 2014;30:559-65.

24. Lennon FE, Mirzapoiazova T, Mambetsariev B, et al. The mu opioid receptor promotes opioid and growth factor-induced proliferation, migration and epithelial mesenchymal transition (emt) in human lung cancer. PLoS One 2014;9:e91577.

25. Chang YC, Liu CL, Chen MJ, et al. Local anesthetics induce apoptosis in human breast tumor cells. Anesth Analg 2014;118:116-24.

26. Lee BM, Rodriguez A, Mena G, et al. Plateletto-lymphocyte ratio and use of nsaids during the perioperative period as prognostic indicators in 
patients with nsclc undergoing surgery. Cancer Control 2016;23:284-94.

27. Tao Y, Li Y, Liu X, et al. Nonsteroidal anti-inflammatory drugs, especially aspirin, are linked to lower risk and better survival of hepatocellular carcinoma: A metaanalysis. Cancer Manag Res 2018;10:2695-709.

28. Takami Y, Eguchi S, Tateishi M, et al. A randomised controlled trial of meloxicam, a cox-2 inhibitor, to prevent hepatocellular carcinoma recurrence after initial curative treatment. Hepatol Int 2016;10:799-806.

29. Cata JP, Guerra CE, Chang GJ, et al. Non-steroidal anti-inflammatory drugs in the oncological surgical population: Beneficial or harmful? A systematic review of the literature. Br J Anaesth 2017;119:750-64.

30. Desmedt C, Demicheli R, Fornili M, et al. Potential benefit of intra-operative administration of ketorolac on breast cancer recurrence according to the patient's body mass index. J Natl Cancer Inst 2018;110:1115-22.
Cite this article as: Wang X, Xie W, Gan S, Wang T, Chen X, Su D, Sun J, Lin J, Wu F, Xu P, Miao C, Yan M, Zhu S, Zhai B, Sun Y, Yu W, Tian J. Effects of general anesthesia versus local anesthesia in primary hepatocellular carcinoma patients presenting for thermal ablation surgery: a multiple center retrospective cohort study with propensity score matching. Ann Transl Med 2020;8(6):277. doi: 10.21037/atm.2020.03.88 
Table S1 Results of univariable Cox regression model analysis on RFS and OS

\begin{tabular}{|c|c|c|c|c|c|c|c|c|c|c|c|c|}
\hline \multirow{3}{*}{ Variables } & \multicolumn{6}{|c|}{ Before PSM } & \multicolumn{6}{|c|}{ After PSM } \\
\hline & \multicolumn{3}{|c|}{ RFS } & \multicolumn{3}{|c|}{ os } & \multicolumn{3}{|c|}{ RFS } & \multicolumn{3}{|c|}{ os } \\
\hline & $\mathrm{HR}$ & $95 \% \mathrm{Cl}$ & $P$ value & $\mathrm{HR}$ & $95 \% \mathrm{Cl}$ & $P$ value & $\mathrm{HR}$ & $95 \% \mathrm{Cl}$ & $P$ value & $\mathrm{HR}$ & $95 \% \mathrm{Cl}$ & $P$ value \\
\hline \multicolumn{13}{|l|}{ Age } \\
\hline$\leq 60$ & 1 & & & 1 & & & 1 & & & 1 & & \\
\hline$>60$ & 1.275 & $1.001-1.623$ & 0.049 & 1.524 & $0.992-2.341$ & 0.054 & 1.146 & $0.862-1.524$ & 0.347 & 1.111 & $0.666-1.853$ & 0.688 \\
\hline \multicolumn{13}{|l|}{ Sex } \\
\hline Male & 1 & & & 1 & & & 1 & & & 1 & & \\
\hline Female & 0.841 & $0.615-1.149$ & 0.277 & 0.933 & $0.541-1.608$ & 0.802 & 0.88 & $0.605-1.279$ & 0.502 & 1.063 & $0.566-1.996$ & 0.85 \\
\hline \multicolumn{13}{|l|}{ ASA score } \\
\hline II & 1 & & & 1 & & & 1 & & & 1 & & \\
\hline III & 1.657 & $1.253-2.191$ & 0 & 1.35 & $0.810-2.251$ & 0.25 & 1.888 & $1.368-2.604$ & 0 & 1.677 & $0.949-2.963$ & 0.075 \\
\hline \multicolumn{13}{|l|}{ Hypertension } \\
\hline No & 1 & & & 1 & & & 1 & & & 1 & & \\
\hline Yes & 1.429 & $1.097-1.861$ & 0.008 & 1.279 & $0.792-2.066$ & 0.315 & 1.161 & $0.837-1.611$ & 0.371 & 1.027 & $0.557-1.894$ & 0.16 \\
\hline Diabetes & & & & & & & & & & & & \\
\hline No & 1 & & & 1 & & & 1 & & & 1 & & \\
\hline Yes & 1.429 & $1.040-1.963$ & 0.028 & 1.102 & $0.610-1.991$ & 0.748 & 1.443 & $0.983-2.118$ & 0.061 & 0.925 & $0.421-2.031$ & 0.846 \\
\hline Cardiopathy & & & & & & & & & & & & \\
\hline No & 1 & & & 1 & & & 1 & & & 1 & & \\
\hline Yes & 1.258 & $0.747-2.118$ & 0.388 & 0.737 & $0.233-2.333$ & 0.603 & 1.201 & $0.565-2.553$ & 0.635 & 0.545 & $0.076-3.934$ & 0.547 \\
\hline Tumor number & & & & & & & & & & & & \\
\hline Solitary & 1 & & & 1 & & & 1 & & & 1 & & \\
\hline Multiple & 1.901 & $1.423-2.540$ & 0 & 1.595 & $0.956-2.661$ & 0.074 & 2.012 & $1.441-2.811$ & 0 & 1.712 & $0.957-3.065$ & 0.07 \\
\hline Cirrhosis & & & & & & & & & & & & \\
\hline No & 1 & & & 1 & & & 1 & & & 1 & & \\
\hline Yes & 1.535 & $1.114-2.114$ & 0.009 & 1.99 & $1.028-3.853$ & 0.041 & 1.701 & $1.165-2.484$ & 0.006 & 2.144 & $0.976-4.708$ & 0.057 \\
\hline $\mathrm{HBV} / \mathrm{HCV}$ infec & & & & & & & & & & & & \\
\hline No & 1 & & & 1 & & & 1 & & & 1 & & \\
\hline Yes & 1.233 & $0.860-1.766$ & 0.255 & 1.054 & $0.559-1.987$ & 0.872 & 1.225 & $0.816-1.839$ & 0.327 & 1.031 & $0.508-2.090$ & 0.934 \\
\hline Adjuvant chem & & & & & & & & & & & & \\
\hline No & 1 & & & 1 & & & 1 & & & 1 & & \\
\hline Yes & 1.825 & $1.404-2.372$ & 0 & 1.337 & $0.811-2.203$ & 0.255 & 1.895 & $1.379-2.606$ & 0 & 1.503 & $0.822-2.748$ & 0.185 \\
\hline AFP & & & & & & & & & & & & \\
\hline$<400 \mathrm{ng} / \mathrm{mL}$ & 1 & & & 1 & & & 1 & & & 1 & & \\
\hline$\geq 400 \mathrm{ng} / \mathrm{mL}$ & 1.347 & $0.978-1.856$ & 0.068 & 1.483 & $0.860-2.558$ & 0.156 & 1.295 & $0.882-1.901$ & 0.187 & 1.424 & $0.742-2.735$ & 0.288 \\
\hline TBIL & & & & & & & & & & & & \\
\hline$<34 \mathrm{mmol} / \mathrm{L}$ & 1 & & & 1 & & & 1 & & & 1 & & \\
\hline$\geq 34 \mathrm{mmol} / \mathrm{L}$ & 1.085 & $0.729-1.616$ & 0.688 & 1.4 & $0.723-2.711$ & 0.318 & 1.211 & $0.776-1.888$ & 0.4 & 1.463 & $0.695-3.076$ & 0.316 \\
\hline ALB & & & & & & & & & & & & \\
\hline$>35 \mathrm{~g} / \mathrm{L}$ & 1 & & & 1 & & & 1 & & & 1 & & \\
\hline$\leq 35 \mathrm{~g} / \mathrm{L}$ & 1.318 & $0.974-1.784$ & 0.073 & 1.977 & $1.224-3.195$ & 0.005 & 1.438 & $1.016-2.035$ & 0.04 & 2.335 & $1.360-4.008$ & 0.002 \\
\hline ALT & & & & & & & & & & & & \\
\hline$<40 \mathrm{U} / \mathrm{L}$ & 1 & & & 1 & & & 1 & & & 1 & & \\
\hline$\geq 40 \mathrm{U} / \mathrm{L}$ & 1.096 & $0.860-1.398$ & 0.458 & 1.223 & $0.794-1.885$ & 0.361 & 1.196 & $0.898-1.592$ & 0.221 & 1.466 & $0.887-2.423$ & 0.135 \\
\hline AST & & & & & & & & & & & & \\
\hline$<40 \mathrm{U} / \mathrm{L}$ & 1 & & & 1 & & & 1 & & & 1 & & \\
\hline$\geq 40 \mathrm{U} / \mathrm{L}$ & 1.094 & $0.860-1.392$ & 0.466 & 1.315 & $0.856-2.018$ & 0.211 & 1.284 & $0.969-1.700$ & 0.081 & 1.631 & $0.991-2.685$ & 0.054 \\
\hline Child-Pugh sta & & & & & & & & & & & & \\
\hline A & 1 & & & 1 & & & 1 & & & 1 & & \\
\hline B & 1.387 & $0.948-2.030$ & 0.092 & 1.851 & $1.004-3.413$ & 0.049 & 1.443 & $0.947-2.199$ & 0.088 & 2.208 & $1.149-4.241$ & 0.017 \\
\hline Tumor size & & & & & & & & & & & & \\
\hline$<3 \mathrm{~cm}$ & 1 & & & 1 & & & 1 & & & 1 & & \\
\hline$\geq 3 \mathrm{~cm}$ & 1.737 & $1.366-2.209$ & 0 & 2.084 & $1.356-3.202$ & 0.001 & 1.872 & $1.414-2.477$ & 0 & 2.12 & $1.285-3.500$ & 0.003 \\
\hline TNM stage & & & & & & & & & & & & \\
\hline I & 1 & & & 1 & & & 1 & & & 1 & & \\
\hline II & 2.154 & $1.638-2.831$ & 0 & 2.263 & $1.431-3.578$ & 0 & 2.269 & $1.646-3.128$ & 0 & 2.244 & $1.306-3.856$ & 0.003 \\
\hline Usage of NSAI & & & & & & & & & & & & \\
\hline No & 1 & & & 1 & & & 1 & & & 1 & & \\
\hline Yes & 1.194 & $0.932-1.529$ & 0.162 & 1.156 & $0.742-1.804$ & 0.521 & 1.108 & $0.825-1.488$ & 0.495 & 1.195 & $0.708-2.017$ & 0.505 \\
\hline Anesthesia me & & & & & & & & & & & & \\
\hline LA & 1 & & & 1 & & & 1 & & & 1 & & \\
\hline GA & 1.47 & $1.153-1.874$ & 0.002 & 1.539 & $0.993-2.386$ & 0.052 & 1.445 & $1.088-1.921$ & 0.011 & 1.614 & $0.964-2.702$ & 0.066 \\
\hline
\end{tabular}

RFS, recurrence-free survival; OS, overall survival; HR, hazard ratio; Cl, confidence interval; ASA, American Society of Anesthesiologists; $\mathrm{HBV}$, hepatitis B virus; $\mathrm{HCV}$, hepatitis C virus; TNM, Clinicopathological stage; AFP, alpha-fetoprotein; TBIL, total bilirubin; ALB, serum albumin; ALT, Alanine transaminase; AST, aspartate aminotransferase; LA, local anaesthesia; GA, general anaesthesia; NSAIDs, Non-Steroidal Anti-inflammatory Drugs. 